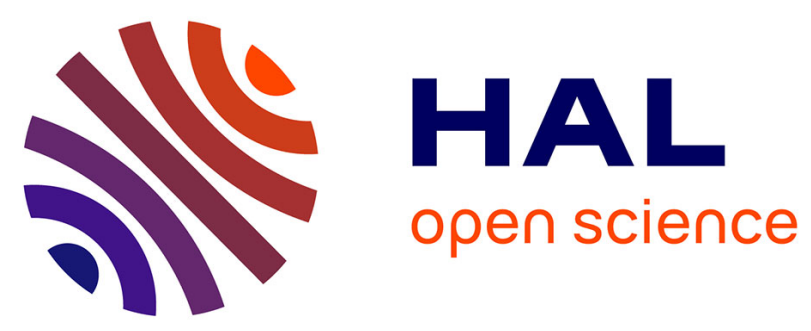

\title{
Synthesis of Isocoumarin via PTSA-Catalyzed Annulation of Diarylalkynes
}

\author{
Gaëlle Le Bras, Abdallah Hamze, Samir Messaoudi, Olivier Provot, \\ Pierre-Benoît Le Calvez, Jean-Daniel Brion, Mouad Alami
}

\section{To cite this version:}

Gaëlle Le Bras, Abdallah Hamze, Samir Messaoudi, Olivier Provot, Pierre-Benoît Le Calvez, et al.. Synthesis of Isocoumarin via PTSA-Catalyzed Annulation of Diarylalkynes. Synthesis: Journal of Synthetic Organic Chemistry, 2008, 2008 (10), pp.1607-1611. 10.1055/s-2008-1072575 . hal-02394596

\section{HAL Id: hal-02394596 https://hal.science/hal-02394596}

Submitted on 4 Dec 2019

HAL is a multi-disciplinary open access archive for the deposit and dissemination of scientific research documents, whether they are published or not. The documents may come from teaching and research institutions in France or abroad, or from public or private research centers.
L'archive ouverte pluridisciplinaire HAL, est destinée au dépôt et à la diffusion de documents scientifiques de niveau recherche, publiés ou non, émanant des établissements d'enseignement et de recherche français ou étrangers, des laboratoires publics ou privés. 


\section{Synthesis of isocoumarin via PTSA-catalyzed annulation of diarylalkynes}

Gaëlle Le Bras, Abdallah Hamze, Samir Messaoudi, Olivier Provot,* Pierre-Benoît Le Calvez, Jean-Daniel Brion and Mouâd Alami*

Univ Paris-Sud, CNRS, BioCIS, UMR 8076, Laboratoire de Chimie Thérapeutique, Faculté de Pharmacie, rue J.B. Clément, Châtenay-Malabry, F-92296, FranceFax: +33-1-46.83.58.28; E-mail: olivier.provot@u-psud.fr and/or mouad.alami@upsud.fr

\begin{abstract}
Toluenesulfonic acid (PTSA) in EtOH was used as a mild acid-catalyst for the annulation of various functionalized diarylalkynes under microwave irradiation. This free-metal process allowed the synthesis of a range of 3-arylsubstituted isocoumarins in good yields.
\end{abstract}

Key words: alkynes, annulations, isocoumarin, isochromene, microwaves activation

Isocoumarin structures are important components in many natural products that exhibit a broad range of biological activitities including antiallergic and antimicrobial, ${ }^{1,2}$ antifungal, ${ }^{3}$ antiinflammatory, ${ }^{4}$ immunomodulatory, ${ }^{5} \quad$ cytotoxic, ${ }^{6}$ and antiangiogenic. ${ }^{7}$ Therefore, a number of methods have been reported in the literature for the synthesis of the isocoumarin ring. ${ }^{8}$ The most common route is undoubtedly the cyclization of 2-(1-alkynyl)benzoic acids/esters under the triple bond activation. ${ }^{9}$ A recent approach to the synthesis of isocoumarins from diarylalkynes using $\mathrm{TFA}^{10}$ as a reagent and solvent prompted us to present our results in this field.

Previously, we reported a new and efficient $p$ toluenesulfonic acid-catalyzed hydration of unsymmetrical aliphatic alkynes in aqueous or alcoholic media. ${ }^{11}$ Interestingly, this new environmentally metalfree procedure has been applied successfully to diarylalkynes to afford regioselectively a series of carbonyl compounds. ${ }^{12}$ Following this work, we were interested to examine this mild and friendly procedure with diarylalkynes bearing an ortho alkoxycarbonyl function on the aromatic ring to give the corresponding 3 -arylisocoumarins. Herein we report the results of this study.

First, we have studied the reaction with diarylalkyne 1a bearing an ortho ethoxycarbonyl function on the aromatic ring as a model substrate. The results, summarized in Table 1, showed that treatment of 1a with a catalytic amount of PTSA $(20 \mathrm{~mol} \%)$ in refluxing EtOH afforded the corresponding isocoumarin $2 \mathbf{a}$ in a good yield but with a prolonged reaction time $(70 \%, 24$ $\mathrm{h}$, entry 1). Next, in the continuation of our work to develop rapid and efficient methodologies, ${ }^{13}$ we choose to promote and accelerate this reaction using microwaves irradiation.

In this way, we were pleased to observe that a reaction time of 30 minutes was sufficient to reach up to $98 \%$ conversion of starting diarylalkyne 1a and isocoumarin 2a was obtained in an excellent yield (89\%, entry 2). As a control experiment, 1a was heated in $\mathrm{EtOH}$ in a sealed tube at $160{ }^{\circ} \mathrm{C}$ for 30 minutes. Comparison of the results obtained using conventional or microwave heating indicated clearly the efficiency of the latter method (89\%, entry 2 vs $30 \%$ entry 3 ). Other carboxyl groups were also examined for this annulation reaction. Interestingly, the yield of $\mathbf{2 a}$ was remarkably increased by switching the ethoxycarbonyl group to a carboxylic acid function (98\%, entry 4$)$. The presence of an amide group on the aromatic ring smoothly affected the yield of this process. In this case, 2a was isolated in a $74 \%$ isolated yield (entry 5) together with $19 \%$ of the 3arylisoquinolin-1-one resulting from the 6-endo-dig nitrogen-cyclization. When $1 \mathbf{d}(\mathrm{R}=\mathrm{CN})$ was heated with PTSA in EtOH under microwave irradiation, 2a was still isolated but in a modest yield (38\%, entry 6).

Table 1. PTSA in EtOH promoted annulation of orthosubstituted arylalkynes $\mathbf{1}$.

\begin{tabular}{llllll} 
& & & & \\
\hline 1 & 1a: $\mathrm{R}=\mathrm{CO}_{2} \mathrm{Et}$ & reflux & 24 & 78 & 70 \\
2 & 1a: $\mathrm{R}=\mathrm{CO}_{2} \mathrm{Et}$ & microwaves & 0.5 & 160 & 89 \\
3 & 1a: $\mathrm{R}=\mathrm{CO}_{2} \mathrm{Et}$ & sealed tube & 0.5 & 160 & 30 \\
4 & 1b: $\mathrm{R}=\mathrm{CO}_{2} \mathrm{H}$ & microwaves & 0.5 & 160 & 98 \\
5 & 1c: $\mathrm{R}=\mathrm{CONH}_{2}$ & microwaves & 0.5 & 160 & $74^{\mathrm{b}}$ \\
6 & 1d: $\mathrm{R}=\mathrm{CN}^{\circ}$ & microwaves & 0.5 & 160 & 38 \\
\hline \hline
\end{tabular}

a. Isolated yield.

b. $19 \%$ of 3-(4-methoxyphenyl)isoquinolin-1-one were isolated.

Using our protocol, we were able to prepare a series of functionalized isocoumarins from various diarylalkynes $\mathbf{1}$ in good yields (Table 2). For practical considerations, we choose to prepare these isocoumarins $\mathbf{2}$ from diarylalkynes bearing an ortho ethoxycarbonyl group (rather than $\mathrm{COOH}$ ), as they are easily available after Sonogashira-Linstrumelle couplings. ${ }^{14}$

As shown in entries 2 and 3, the presence of a methoxy group on the ortho or the meta position did not affected significantly the yield of this annulation process. A similar result was obtained starting from $\mathbf{1 g}, \mathbf{1 h}$ or $\mathbf{1 i}$ which provided the 3-arylisocoumarins 2 d-f in a satisfactory isolated yield (entry 4-6). The presence of a nitro group on the aromatic ring did not interfere with 
Table 2. One pot synthesis of 3-aryl-isocoumarins 2 from diarylalkynes 1

\begin{tabular}{|c|c|c|c|c|c|c|}
\hline Entry & Alkyne 1 & & Time (h) & Isocoumarin 2 & & Yield $^{\mathrm{a}}(\%)$ \\
\hline 1 & & 1a & 0.5 & & $2 a$ & 89 \\
\hline 2 & & 1e & 1 & & $2 b$ & 70 \\
\hline 3 & & 1f & 1 & & $2 c$ & 64 \\
\hline 4 & & & 1 & & 2d & 62 \\
\hline 5 & & $1 \mathrm{~h}$ & 1 & & $2 e$ & 60 \\
\hline 6 & & $\mathbf{1 i}$ & 2 & & $2 f$ & 67 \\
\hline 7 & & $\mathbf{1 j}$ & 1 & & $2 \mathrm{~g}$ & 61 \\
\hline 8 & & $1 \mathbf{k}$ & 2 & & $2 h$ & 85 \\
\hline 9 & & 11 & 2 & & $2 \mathbf{i}$ & 59 \\
\hline 10 & & $1 \mathrm{~m}$ & 2 & & $2 \mathrm{j}$ & $88^{\mathrm{b}}$ \\
\hline
\end{tabular}

${ }^{a}$ Isolated yield.

${ }^{\mathrm{b}}$ obtained as an inseparable 65/35 mixture with the Markovnikov ketone resulting from the triple bond hydration. 
the outcome of this process as demonstrated with diarylalkyne 1j (entry 7, 61\%). Similarly, the arylnaphtlyl alkyne 1k was successfully transformed into to the isocoumarin $\mathbf{2 h}$ in an excellent $85 \%$ yield (entry 8). Finally, when replacing the phenyl substituent by a pyridine ring, this methodology was still efficient and the corresponding isocoumarin derivative $\mathbf{2} \mathbf{i}$ was obtained in a satisfactory isolated yield and with a reasonable reaction time (entry 9, $2 \mathrm{~h}, 59 \%$ ). Interestingly, we observed that our protocol was still efficient with the aliphatic arylalkyne $\mathbf{1 m}$. In that case, 3-pentylisocoumarin $\mathbf{2 j}$ was obtained in a good yield (entry 10, 82\%) suggesting that the presented protocol could be applied successfully to a range of aliphatic arylalkynes.

To show the high synthetic potential of this protocol, we have tested it with the diarylalkyne $1 \mathbf{n}$ bearing a nucleophilic ortho hydroxymethyl substituent in place of the ethoxycarbonyl group (Scheme 1). We were pleased to observe that after stirring for 30 minutes at $120{ }^{\circ} \mathrm{C}$ under microwave heating, the annulation proceeded effectively to give in good yield the desired isochromene $\mathbf{2 k}$.

Scheme 1. Isochromene $\mathbf{2 k}$ via PTSA-catalyzed annulation of $\mathbf{1 n}$.

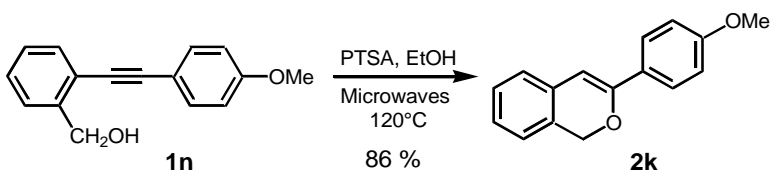

In conclusion, a novel and reliable procedure for the synthesis of isocoumarins was achieved via PTSAcatalyzed annulation of ortho substituted arylalkynes in $\mathrm{EtOH}$ under microwave irradiation. This metalfree procedure is characterized by the mildness of acidic conditions, short reaction times and good yields. The synthesis of other heterocycles such as isoquinolines and isothiochromenes from their corresponding ortho substituted benzylamine or thiol derivatives respectively is currently under investigation and will be presented in due course.

IR spectra were recorded on a Perkin-Elmer 841 spectrometer. ${ }^{1} \mathrm{H}$ and ${ }^{13} \mathrm{C}$ NMR spectra were measured with a Bruker Avance $300\left(300 \mathrm{MHz}\right.$ and $75 \mathrm{MHz}$, for ${ }^{1} \mathrm{H}$ and ${ }^{13} \mathrm{C}$, respectively). ${ }^{1} \mathrm{H}$ chemical shifts are reported in ppm from an internal standard TMS or residual chloroform (7.27 ppm). ${ }^{13} \mathrm{C}$ chemical shifts are reported from the central peak of deuteriochloroform (77.1 ppm). ESI mass spectra were obtained with a LCT Waters-Micromass spectrometer Elemental analyses were performed with a Perkin-Elmer 240 analyzer. Melting points were recorded on Büchi B-450 apparatus and are uncorrected. Analytical TLC were performed on Merck precoated silica gel 60F plates. Merck silica gel 60 (230-400 mesh) was used for column chromatography. All microwave experiments were performed using an Emrys Optimizer in 2-5 $\mathrm{mL}$ pyrex reaction vessels. Each contained a Teflon stir bar and Teflon coated reaction vessel cap.

Typical procedure: To an Emrys Optimizer 2-5 mL pyrex reaction vessel were added diarylalkyne $(0.5 \mathrm{mmol})$, PTSA $(0.01 \mathrm{mmol})$, in EtOH $(2.5 \mathrm{~mL})$. The reaction vessel was then placed in the Emrys Optimizer and exposed to microwave irradiation according to the following specifications: temperature: $160^{\circ} \mathrm{C}$, time (see Table 2), fixed hold time: on, sample absorption: high, pre-stirring: $60 \mathrm{~s}$. After cooling to room temperature, $\mathrm{H}_{2} \mathrm{O}(3 \mathrm{~mL})$ was added to the crude and the mixture was extracted with EtOAc $(3 \times 2 \mathrm{~mL})$. Organic layers were then washed with an aqueous saturated $\mathrm{NH}_{4} \mathrm{Cl}$ solution, dried and concentrated. The crude mixture was then purified by column chromatography on silica gel.

\section{3-(4-Methoxyphenyl)isocoumarin 2a:}

White solid; mp $111-113^{\circ} \mathrm{C}$.

IR (neat): 2999, 2844, 1957, 1734, 1632, 1601, 1575, 1562, $1512,1480,1457,1442,1420,1344,1309,1287,1260,1235$, $1200,1175,1114,1064,1020,925,887,835,790 \mathrm{~cm}^{-1}$.

${ }^{1} \mathrm{H}$ NMR $\left(300 \mathrm{MHz}, \mathrm{CDCl}_{3}\right): \delta 3.90(\mathrm{~s}, 3 \mathrm{H}), 6.86(\mathrm{~s}, 1 \mathrm{H}), 7.00$ $(\mathrm{d}, 2 \mathrm{H}, J=9.0 \mathrm{~Hz}), 7.44-7.50(\mathrm{~m}, 2 \mathrm{H}), 7.75(\mathrm{t}, 1 \mathrm{H}, J=7.8 \mathrm{~Hz})$, $7.86(\mathrm{~d}, 2 \mathrm{H}, J=9.0 \mathrm{~Hz}), 8.32(\mathrm{~d}, 1 \mathrm{H}, J=7.8 \mathrm{~Hz})$.

${ }^{13} \mathrm{C}$ NMR $\left(75 \mathrm{MHz}, \mathrm{CDCl}_{3}\right): \delta 55.4\left(\mathrm{CH}_{3}\right), 100.2(\mathrm{CH}), 114.3$ $(2 \mathrm{CH}), 120.2(\mathrm{C}), 124.4(\mathrm{C}), 125.7(\mathrm{CH}), 126.8(2 \mathrm{CH}), 127.7$ $(\mathrm{CH}), 129.6(\mathrm{CH}), 134.8(\mathrm{CH}), 137.9(\mathrm{C}), 153.7(\mathrm{C}), 161.0(\mathrm{C})$, $162.6(\mathrm{C})$.

Anal. Calcd for $\mathrm{C}_{16} \mathrm{H}_{12} \mathrm{O}_{3}$ : C, 76.18; H, 4.79. Found: C, 76.00; H, 4.67 .

MS $m / z(\mathrm{ES}+) 275.0\left(\mathrm{M}+\mathrm{Na}^{+}\right)$.

\section{3-(2-Methoxyphenyl)isocoumarin 2b:}

White solid; mp $115-117{ }^{\circ} \mathrm{C}$.

IR (neat): 3117, 3011, 2978, 2843, 1716, 1621, 1596, 1575, $1562,1483,1460,1435,1366,1336,1313,1277,1252,1178$, $1165,1130,1108,1071,1015,963,944,924,887,854, \mathrm{~cm}^{-1}$.

${ }^{1} \mathrm{H} \mathrm{NMR}\left(300 \mathrm{MHz}, \mathrm{CDCl}_{3}\right): \delta 4.00$ (s, 3H), 7.00-7.14 (m, 2H), 7.37-7.44 (m, 2H), $7.52(\mathrm{~d}, 2 \mathrm{H}, J=7.6 \mathrm{~Hz}), 7.70(\mathrm{td}, 1 \mathrm{H}, J=$ $7.9 \mathrm{~Hz}, J=1.2 \mathrm{~Hz}), 8.00(\mathrm{~d}, 1 \mathrm{H}, J=7.9 \mathrm{~Hz}), 8.33(\mathrm{~d}, 1 \mathrm{H}, J=$ $7.9 \mathrm{~Hz})$.

${ }^{13} \mathrm{C}$ NMR $\left(75 \mathrm{MHz}, \mathrm{CDCl}_{3}\right): \delta 55.7\left(\mathrm{CH}_{3}\right), 107.1(\mathrm{CH}), 111.4$ $(\mathrm{CH}), 120.7(\mathrm{CH}), 120.9(\mathrm{C}), 126.3(\mathrm{CH}), 128.0(\mathrm{CH}), 128.9$ $(\mathrm{CH}), 129.4(\mathrm{CH}), 130.8(\mathrm{C}), 134.6(\mathrm{CH}), 138.1(\mathrm{CH}), 145.1$ (C), $150.5(\mathrm{C}), 157.3(\mathrm{C}), 162.6(\mathrm{C})$.

Anal. Calcd for $\mathrm{C}_{16} \mathrm{H}_{12} \mathrm{O}_{3}$ : C, 76.18; H, 4.79. Found: C, 76.03; H, 4.66 .

\section{3-(3,4,5-Trimethoxyphenyl)isocoumarin $2 \mathrm{c}$ :}

Beige solid; mp $171{ }^{\circ} \mathrm{C}$.

IR (neat): 1716, 1635, 1580, 1501, 1416, 1240, 1170, 1119, $765,750,684 \mathrm{~cm}^{-1}$. 
${ }^{1} \mathrm{H}$ NMR (300 MHz, $\left.\mathrm{CDCl}_{3}\right): \delta 3.83$ (s, 3H), 3.88 (s, 6H), 6.80 (s, 1H), $7.00(\mathrm{~s}, 2 \mathrm{H}), 7.37-7.44(\mathrm{~m}, 2 \mathrm{H}), 7.63$ (dt, 1H, $J=7.6$ $\mathrm{Hz}, J=1.3 \mathrm{~Hz}), 8.22(\mathrm{~d}, 1 \mathrm{H}, J=4.4 \mathrm{~Hz})$.

${ }^{13} \mathrm{C} \mathrm{NMR}\left(75 \mathrm{MHz}, \mathrm{CDCl}_{3}\right): \delta 56.2\left(2 \mathrm{CH}_{3}\right), 60.8\left(\mathrm{CH}_{3}\right), 101.5$ $(\mathrm{CH}), 102.7(2 \mathrm{CH}), 120.3(\mathrm{C}), 125.8(\mathrm{CH}), 127.5(\mathrm{C}), 128.0$ $(\mathrm{CH}), 129.7(\mathrm{CH}), 134.9(\mathrm{CH}), 137.5(\mathrm{C}), 139.9(\mathrm{C}), 153.4(\mathrm{C})$, 153.5 (2 C), 162.2 (C).

Anal. Calcd for $\mathrm{C}_{18} \mathrm{H}_{16} \mathrm{O}_{5}$ : C, 69.22; H, 5.16. Found: C, 69.15; H, 5.12 .

MS $m / z(\mathrm{ES}+) 335.0\left(\mathrm{M}+\mathrm{Na}^{+}\right)$.

\section{3-(4-Methylphenyl)isocoumarin 2d:}

White solid; $\mathrm{mp} 108-110^{\circ} \mathrm{C}$.

IR (neat): 2920, 1776, 1729, 1629, 1607, 1562, 1510, 1482, $1455,1343,1277,1197,1187,1065,845,814 \mathrm{~cm}^{-1}$.

${ }^{1} \mathrm{H}$ NMR (300 MHz, $\left.\mathrm{CDCl}_{3}\right): \delta 2.33(\mathrm{~s}, 3 \mathrm{H}), 6.83(\mathrm{~s}, 1 \mathrm{H}), 7.15-$ $7.23(\mathrm{~m}, 2 \mathrm{H}), 7.36-7.44(\mathrm{~m}, 2 \mathrm{H}), 7.60(\mathrm{t}, 1 \mathrm{H}, J=8.3 \mathrm{~Hz}), 7.70$ $(\mathrm{d}, 2 \mathrm{H}, J=8.3 \mathrm{~Hz}), 8.23(\mathrm{~d}, 1 \mathrm{H}, J=8.3 \mathrm{~Hz})$.

${ }^{13} \mathrm{C}$ NMR $\left(75 \mathrm{MHz}, \mathrm{CDCl}_{3}\right): \delta 21.5\left(\mathrm{CH}_{3}\right), 101.0(\mathrm{CH}), 120.5$ (C), $125.3(2 \mathrm{CH}), 125.9(\mathrm{CH}), 128.0(\mathrm{CH}), 129.3(\mathrm{C}), 129.7$ $(2 \mathrm{CH}), 129.8(\mathrm{CH}), 134.9(\mathrm{CH}), 137.8(\mathrm{C}), 140.4(\mathrm{C}), 154.0$ (C), 162.5 (C).

Anal. Calcd for $\mathrm{C}_{16} \mathrm{H}_{12} \mathrm{O}_{2}$ : C, 81.34; H, 5.12. Found: C, 81.25; H, 5.08 .

\section{3-(4-Aminophenyl)isocoumarin 2e:}

Yellow solid. mp $158^{\circ} \mathrm{C}$.

IR (neat): 3532-3482, 1705, 1601, 1524, 1072, 812, $748 \mathrm{~cm}^{-1}$.

${ }^{1} \mathrm{H}$ NMR (300 MHz, $\left.\mathrm{CDCl}_{3}\right) \square: \delta 3.78$ (bs, 2H), 6.49 (d, 2H, $J=$ $8.7 \mathrm{~Hz}), 6.59(\mathrm{~s}, 1 \mathrm{H}), 7.29-7.23(\mathrm{~m}, 2 \mathrm{H}), 7.51(\mathrm{t}, 1 \mathrm{H}, J=7.8$ $\mathrm{Hz},), 7.56(\mathrm{~d}, 2 \mathrm{H}, J=8.7 \mathrm{~Hz}), 8.11(\mathrm{~d}, 1 \mathrm{H}, J=7.7 \mathrm{~Hz})$.

${ }^{13} \mathrm{C} \mathrm{NMR}\left(75 \mathrm{MHz}, \mathrm{CDCl}_{3}\right): \delta 98.5(\mathrm{CH}), 112.4(2 \mathrm{CH}), .119 .7$ (C), $120.4(\mathrm{C}), 125.4(\mathrm{CH}), 126.7(2 \mathrm{CH}), 127.0(\mathrm{CH}), 129.5$ (CH), $134.7(\mathrm{CH}), 138.5(\mathrm{C}), 149.8(\mathrm{C}), 154.6(\mathrm{C}), 162.8(\mathrm{C})$

Anal. Calcd for $\mathrm{C}_{15} \mathrm{H}_{11} \mathrm{NO}_{2}$ : C, 75.94; N, 5.90; H, 4.67. Found: C, 75.61; N, 5.74; H, 4.47.

MS $m / z(\mathrm{ES}+) 238.0\left(\mathrm{M}+\mathrm{H}^{+}\right)$

\section{7-Methoxy-3-(4-methoxyphenyl)isocoumarin 2f:}

White solid; mp $144-146^{\circ} \mathrm{C}$.

IR (neat): 2964, 2839, 1717, 1632, 1602, 1573, 1562, 1510, 1496, 1454, 1440, 1419, 1352, 1290, 1256, 1178, 1163, 1119, $1067,1024,935,889,867,850,837,813 \mathrm{~cm}^{-1}$.

${ }^{1} \mathrm{H}$ NMR (300 MHz, $\left.\mathrm{CDCl}_{3}\right): \delta 3.88(\mathrm{~s}, 3 \mathrm{H}), 3.94(\mathrm{~s}, 3 \mathrm{H}), 6.83$ $(\mathrm{s}, 1 \mathrm{H}), 7.00(\mathrm{~d}, 2 \mathrm{H}, J=8.9 \mathrm{~Hz}), 7.32(\mathrm{dd}, 1 \mathrm{H}, J=8.6 \mathrm{~Hz}, J=$ $2.6 \mathrm{~Hz}), 7.42(\mathrm{~d}, 1 \mathrm{H}, J=8.6 \mathrm{~Hz}), 7.73(\mathrm{~d}, 1 \mathrm{H}, J=2.6 \mathrm{~Hz}), 7.82$ $(\mathrm{d}, 2 \mathrm{H}, J=8.9 \mathrm{~Hz})$.

${ }^{13} \mathrm{C} \mathrm{NMR}\left(75 \mathrm{MHz}, \mathrm{CDCl}_{3}\right): \delta 55.5\left(\mathrm{CH}_{3}\right), 55.9\left(\mathrm{CH}_{3}\right), 100.2$ $(\mathrm{CH}), 110.0(\mathrm{CH}), 114.3(2 \mathrm{CH}), 121.3(\mathrm{C}), 124.9(\mathrm{CH}), 126.6$ $(2 \mathrm{CH}), 127.4(\mathrm{CH}), 131.5(\mathrm{C}), 131.8(\mathrm{C}), 134.2(\mathrm{C}), 159.4(\mathrm{C})$, $160.9(\mathrm{C})$.
Anal. Calcd for $\mathrm{C}_{17} \mathrm{H}_{14} \mathrm{O}_{4}$ : C, 72.33; H, 5.00. Found: C, 72.27; H, 4.97.

\section{3-(4-Methoxyphenyl)-7-nitro-isocoumarin 2g:}

Yellow solid. mp $204{ }^{\circ} \mathrm{C}$

IR (neat): 1729, 1599, 1481, 1336, 1178, 1093, $829 \mathrm{~cm}^{-1}$.

${ }^{1} \mathrm{H}$ NMR $\left(300 \mathrm{MHz}, \mathrm{CDCl}_{3}\right): \delta 3.88$ (s, 3H), 6.92 (s, 1H), 7.00 $(\mathrm{d}, 2 \mathrm{H}, J=9.0 \mathrm{~Hz}), 7.60(\mathrm{~d}, 1 \mathrm{H}, J=8.6 \mathrm{~Hz}), 7.86(\mathrm{~d}, 2 \mathrm{H}, J=$ $9.0 \mathrm{~Hz}), 8.42(\mathrm{dd}, 1 \mathrm{H}, J=8.6 \mathrm{~Hz}, J=2.4 \mathrm{~Hz}), 9.12(\mathrm{~d}, 1 \mathrm{H}, J=$ $2.3 \mathrm{~Hz}$ ).

${ }^{13} \mathrm{C} \mathrm{NMR}\left(75 \mathrm{MHz}, \mathrm{CDCl}_{3}\right): \delta 55.5\left(\mathrm{CH}_{3}\right), 99.0(\mathrm{CH}), 114.5(2$ $\mathrm{CH}), 120.1(\mathrm{C}), 123.3(\mathrm{C}), 125.9(\mathrm{CH}), 126.8(\mathrm{CH}), 127.5(2$ $\mathrm{CH}), 129.0(\mathrm{CH}), 143.0(\mathrm{C}), 146.3(\mathrm{C}), 157.3(\mathrm{C}), 160.6(\mathrm{C})$, $162.1(\mathrm{C})$.

Anal. Calcd for $\mathrm{C}_{16} \mathrm{H}_{11} \mathrm{NO}_{5}$ : C, 64.65; N, 4.71; H, 3.73. Found: C, 64.45; N, 4.58; H, 3.68.

MS $m / z(\mathrm{ES}+) 298.0\left(\mathrm{M}+\mathrm{H}^{+}\right)$.

\section{3-Naphthalen-1-yl-isocoumarin $2 \mathrm{~h}$ :}

White solid; mp 120-122 ${ }^{\circ} \mathrm{C}$.

IR (neat): 3091, 3042, 1938, 1715, 1638, 1606, 1566, 1508, 1486, 1454, 1396, 1352, 1310, 1241, 1200, 1178, 1154, 1116, $1065,1023,992,956,922,881 \mathrm{~cm}^{-1}$.

${ }^{1} \mathrm{H}$ NMR (300 MHz, $\left.\mathrm{CDCl}_{3}\right): \delta 6.80(\mathrm{~s}, 1 \mathrm{H}), 7.47-7.61(\mathrm{~m}, 5 \mathrm{H})$, 7.70-7.81 (m, 2H), 7.86-8.00 (m, 2H), 8.21-8.30 (d, $1 \mathrm{H}, J=9.7$ $\mathrm{Hz}), 8.40(\mathrm{~d}, 1 \mathrm{H}, J=7.4 \mathrm{~Hz})$.

${ }^{13} \mathrm{C}$ NMR (75 MHz, $\left.\mathrm{CDCl}_{3}\right): \delta 107.1(\mathrm{CH}), 120.6(\mathrm{C}), 125.0$ $(\mathrm{CH}), 125.1(\mathrm{CH}), 125.9(\mathrm{CH}), 126.3(\mathrm{CH}), 127.1(\mathrm{CH}), 127.7$ $(\mathrm{CH}), 128.4(\mathrm{CH}), 128.6(\mathrm{CH}), 129.7(\mathrm{CH}), 130.6(\mathrm{CH}), 130.8$ (C), 133.8 (2 C), 134.9 (CH), 137.5 (C), 154.7 (C), 162.6 (C).

Anal. Calcd for $\mathrm{C}_{19} \mathrm{H}_{12} \mathrm{O}_{2}$ : C, 83.81; H, 4.44. Found: C, 83.60; H, 4.27 .

\section{3-Pyridin-2-yl-isocoumarin 2i:}

Yellow solid. mp $109-111^{\circ} \mathrm{C}$.

IR (neat): 3059, 3011, 1963, 1737, 1641, 1600, 1582, 1568, $1472,1453,1431,1346,1316,1282,1232,1183,1153,1075$, $1012,990,932,899 \mathrm{~cm}^{-1}$.

${ }^{1} \mathrm{H}$ NMR $\left(300 \mathrm{MHz}, \mathrm{CDCl}_{3}\right): \delta$ 7.47-7.65 $(\mathrm{m}, 1 \mathrm{H}), 7.72-7.88$ (m, 2H), 7.90-8.14 ( m, 3H), $8.30(\mathrm{~d}, 1 \mathrm{H}, J=8.0 \mathrm{~Hz}), 8.54-8.63$ $(\mathrm{m}, 1 \mathrm{H}), 8.90-8.96(\mathrm{~m}, 1 \mathrm{H})$.

${ }^{13} \mathrm{C}$ NMR (75 MHz, $\left.\mathrm{CDCl}_{3}\right): \delta 103.8(\mathrm{CH}), 120.0(\mathrm{CH}), 124.2$ (C), $126.8(\mathrm{CH}), 127.0(\mathrm{CH}), 128.7(\mathrm{CH}), 129.8(\mathrm{CH}), 132.1$ (C), $135.0(\mathrm{CH}), 137.1(\mathrm{CH}), 145.5(\mathrm{C}), 149.7(\mathrm{CH}), 152.2(\mathrm{C})$, $168.7(\mathrm{C})$.

Anal. Calcd for $\mathrm{C}_{14} \mathrm{H}_{9} \mathrm{NO}_{2}$ : C, 75.33; N, 6.27; H, 4.06. Found: C, 75.29; N, 6.21; H, 3.97.

\section{3-nPentyl-isocoumarin $\mathbf{2 j}$}

Yellow oil

IR (neat): 2927, 1729, 1657, 1342, 1287, $1108 \mathrm{~cm}^{-1}$.

${ }^{1} \mathrm{H}$ NMR $\left(300 \mathrm{MHz}, \mathrm{CDCl}_{3}\right): \delta 0.88-0.95(\mathrm{t}, 3 \mathrm{H}, J=7.8 \mathrm{~Hz})$, 1.05-1.40 (m, 6H), 1.51-1.70 (m, 2H), $6.20(\mathrm{~s}, 1 \mathrm{H}), 7.38(\mathrm{~d}, 1 \mathrm{H}$, 
$J=7.8 \mathrm{~Hz}), 7.42(\mathrm{t}, 1 \mathrm{H}, J=7.8 \mathrm{~Hz}), 7.51(\mathrm{t}, 1 \mathrm{H}, J=7.8 \mathrm{~Hz})$, $8.17(\mathrm{~d}, 1 \mathrm{H}, J=7.8 \mathrm{~Hz})$.

${ }^{13} \mathrm{C}$ NMR $\left(75 \mathrm{MHz}, \mathrm{CDCl}_{3}\right): \delta 14.3\left(\mathrm{CH}_{3}\right), 22.5\left(\mathrm{CH}_{2}\right), 26.6$ $\left(\mathrm{CH}_{2}\right), 31.2\left(\mathrm{CH}_{2}\right), 33.5\left(\mathrm{CH}_{2}\right), 102.9(\mathrm{CH}), 120.1(\mathrm{C}), 125.4$ $(\mathrm{CH}), 127.8(\mathrm{CH}), 129.9(\mathrm{CH}), 134.4(\mathrm{CH}), 136.7(\mathrm{C}), 158.3$ (C), $167.1(\mathrm{C})$.

Anal. Calcd for $\mathrm{C}_{14} \mathrm{H}_{16} \mathrm{O}_{2}$ : C, 77.75; H, 7.46. Found: C, 77.48; H, 7.27.

\section{3-(4-Methoxyphenyl)-1H-isochromene 2k:}

White solid; mp $126-128^{\circ} \mathrm{C}$.

IR (neat): 1602, 1501, 1443, 1250, 1170, 1114, 1025, 803, 720 $\mathrm{cm}^{-1}$.

${ }^{1} \mathrm{H} \mathrm{NMR}\left(300 \mathrm{MHz}, \mathrm{CDCl}_{3}\right): \delta 3.54$ (s, 3H), $5.20(\mathrm{~s}, 2 \mathrm{H}), 6.35$ (s, 1H), $6.91(\mathrm{~d}, 2 \mathrm{H}, J=8.4 \mathrm{~Hz}), 7.08-7.26(\mathrm{~m}, 4 \mathrm{H}), 7.68$ (d, $2 \mathrm{H}, J=8.4 \mathrm{~Hz}$ ).

${ }^{13} \mathrm{C}$ NMR $\left(75 \mathrm{MHz}, \mathrm{CDCl}_{3}\right): \delta 55.3\left(\mathrm{CH}_{3}\right), 69.0\left(\mathrm{CH}_{2}\right), 99.5$ $(\mathrm{CH}), 113.7(2 \mathrm{CH}), 123.5(\mathrm{CH}), 126.0(\mathrm{CH}), 126.6(\mathrm{CH})$, 126.9 (2 CH), $127.8(\mathrm{C}), 128.2(\mathrm{C}), 129.1(\mathrm{CH}), 132.3(\mathrm{C})$, 154.0 (C), 160.3 (C).

Anal. Calcd for $\mathrm{C}_{16} \mathrm{H}_{14} \mathrm{O}_{2}: \mathrm{C}, 80.65$; H, 5.92. Found: C, 80.51; $\mathrm{H}, 5.84$.

$\mathrm{MS} m / z(\mathrm{ES}+) 239.0\left(\mathrm{M}+\mathrm{H}^{+}\right)$.

\section{Acknowledgment}

The CNRS is gratefully thanked for support of this research. We also thank the Servier Group for a doctoral fellowship to G. L.B.

\section{References}

(1) Matsuda, H.; Shimoda, H.; Yoshikawa, M. Bioorg. Med. Chem. 1999, 7, 1445-1450.

(2) Yoshikawa, M.; Harada, E.; Naitoh, Y.; Inoue, K.; Matsuda, H.; Shimoda, H.; Yamahara, J.; Murakami, N. Chem. Pharm. Bull. 1994, 42, 2225-2230.

(3) Nozawa, K.; Yamada, M.; Tsuda, Y.; Kawai, K.; Nakajima, S. Chem. Pharm. Bull. 1981, 29, 2689-2691.

(4) Furuta, T.; Fukuyama, Y.; Asakawa, Y. Phytochemistry 1986, 25, 517-520.

(5) Matsuda, H.; Shimoda, H.; Yamahara, J.; Yoshikawa, M. Bioorg. Med. Chem. Lett. 1998, 8, 215-220.

(6) Whyte, A. C.; Gloer, J. B.; Scott, J. A.; Mallock, D. J. Nat. Prod. 1996, 59, 765-769.

(7) Lee, J. H.; Park, Y. J.; Kim, H. S.; Hong, Y. S.; Kim, K.W.; Lee, J. J. J. Antibiot. 2001, 54, 463-466.

(8) For a review, see: Napolitano, E. Org. Prep. Proced. Int. 1997, 29, 631-664.

(9) For some recent examples, see: (a) Cherry, K.; Parrain, J.L.; Thibonnet, J.; Duchêne, A.; Abarbri, M. J. Org. Chem. 2005, 70, 6669-6675. (b) Woon, E.C.; Dhami, A.; Mahon, M. F.; Threadgill, M.D. Tetrahedron 2006, 62, 4829-4837. (c) Uchiyama, M.; Ozawa, H.; Takuma, K.;
Matsumoto, Y.; Yonehara, M.; Hiroya, K.; Sakamoto, T. Org. Lett. 2006, 8, 5517-5520. (d) Marchal, E.; Uriac, P.; Legouin, B.; Toupet, L.; van de Weghe, P. Tetrahedron 2007, 63, 9979-9990.

(10) Hellal, M.; Bourguignon, J.-J.; Bihel, F. Tetrahedron Lett. 2008, 49, 62-65.

(11) Olivi, N.; Thomas, E.; Peyrat, J.-F.; Alami, M.; Brion, J.D. Synlett 2004, 2175-2179.

(12) Le Bras, G.; Provot, O.; Peyrat, J.-F.; Alami, M.; Brion, J.-D. Tetrahedron, Lett. 2006, 47, 5497-5501.

(13) (a) Bekaert, A.; Provot, O.; Rasolojaona, O.; Peyrat, J.-F.; Alami, M.; Brion, J.-D. Tetrahedron Lett. 2005, 46, 4187-4191. (b) Le Bras, G.; Provot, O.; Bekaert, A.; Peyrat, J.-F.; Alami, M.; Brion, J.-D. Synthesis 2006, 1537-1541. (c) L'Hermite, N.; Giraud, A.; Provot, O.; Peyrat, J.-F.; Alami, M.; Brion, J.-D. Tetrahedron 2006, 62, 11994-12002. (d) Giraud, A.; Provot, O.; Peyrat, J.F.; Alami, M.; Brion, J.-D. Tetrahedron 2006, 62, 7667-7673.

(14) (a) Sonogashira, K.; Tohda, Y.; Hagihara, N. Tetrahedron Lett. 1975, 4467-4470. (b) Alami, M.; Ferri, F.; Linstrumelle, G. Tetrahedron Lett. 1993, 34, 6403-6406. 
Synthesis of isocoumarin via PTSA-catalyzed annulation of diarylalkynes<smiles>[R][R]([H])=CC(=O)OCC</smiles><smiles>[R]c1ccc2cc(Br)oc(=O)c2c1</smiles> 
Table 1. PTSA in EtOH promoted annulation of ortho -substituted arylalkynes $\mathbf{1 .}$

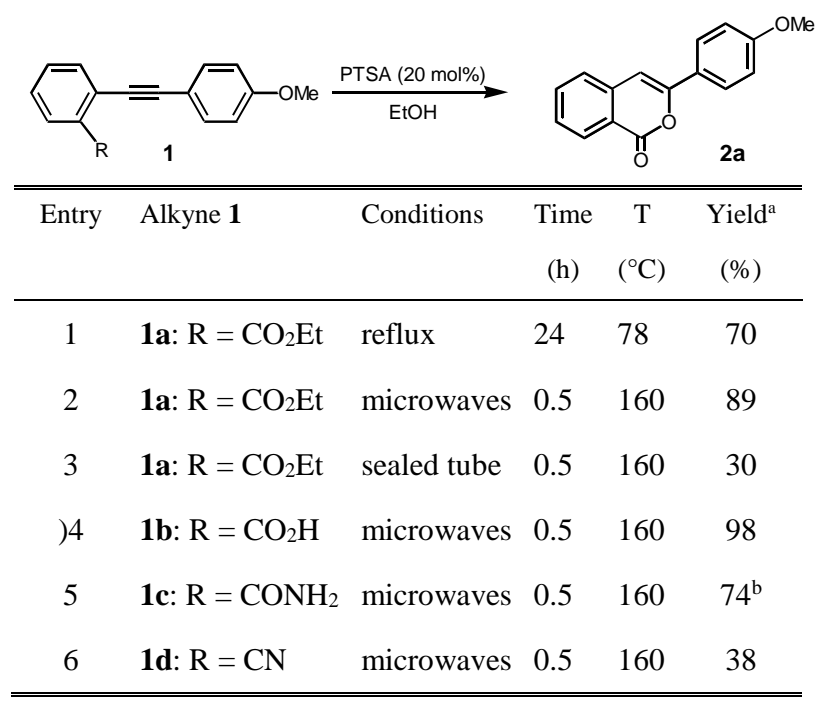

a. Isolated yield.

b. $19 \%$ of 3-(4-methoxyphenyl)isoquinolin-1-one were isolated. 
Table 2. One pot synthesis of 3-aryl-isocoumarins 2 from diarylalkynes 1
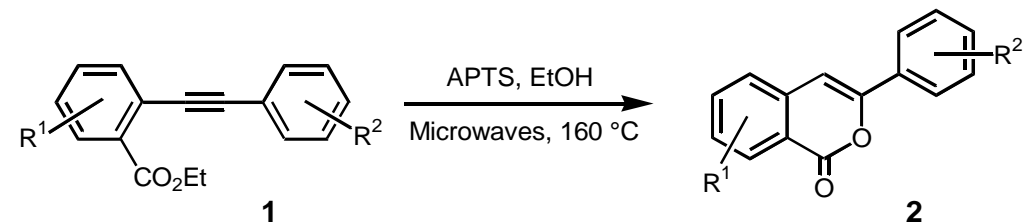

\begin{tabular}{|c|c|c|c|c|c|c|}
\hline Entry & Alkyne 1 & & Time (h) & Isocoumarin 2 & & Yield $^{\mathrm{a}}(\%)$ \\
\hline 1 & & $1 \mathbf{a}$ & 0.5 & & $\mathbf{2 a}$ & 89 \\
\hline 2 & & $\mathbf{1 e}$ & 1 & & $2 b$ & 70 \\
\hline 3 & & 1f & 1 & & $2 c$ & 64 \\
\hline 4 & & $1 \mathrm{~g}$ & 1 & & $2 d$ & 62 \\
\hline 5 & & 1h & 1 & & $2 e$ & 60 \\
\hline 6 & & $\mathbf{1 i}$ & 2 & & $2 f$ & 67 \\
\hline 7 & & $1 \mathrm{j}$ & 1 & & $2 \mathrm{~g}$ & 61 \\
\hline 8 & & $1 k$ & 2 & & $2 h$ & 85 \\
\hline 9 & & 11 & 2 & & $2 \mathrm{i}$ & 59 \\
\hline 10 & & $1 \mathrm{~m}$ & 2 & & $2 \mathbf{j}$ & $88^{\mathrm{b}}$ \\
\hline
\end{tabular}

${ }^{\mathrm{a}}$ Isolated yield.

${ }^{\mathrm{b}}$ obtained as an inseparable 65/35 mixture with the Markovnikov ketone resulting from the triple bond hydration 
Scheme 1. Isochromene 2k via PTSA-catalyzed annulation of $\mathbf{1 n}$.<smiles>COc1ccc(C2=Cc3cc(ccc3O)COC2c2ccccc2)cc1</smiles> 
Scheme 1. Isochromene $2 \mathbf{k}$ via PTSA-catalyzed annulation of $\mathbf{1 n}$.<smiles>COc1ccc(C2=Cc3cc(ccc3O)COC2c2ccccc2)cc1</smiles> 